\title{
Coverage of Generalized Chess Boards \\ by Randomly Placed Rooks
}

\author{
by \\ Leo Katz ${ }^{1}$ \\ and Milton Sobe ${ }^{2}$ \\ Michigan State University \\ University of Minnesota \\ Technical Report No. 144
}

\author{
University of Minnesota \\ Minneapolis, Minnesota
}

September 1970

\footnotetext{
Research supported by NSF Grant GP-11021.

Research supported by NSF Grant GP -13484 .
} 
Introduction.

At a recent colloquium on combinatorial structures, Kamps and van Lint [2] presented a paper on the minimal number of rooks $\sigma(n, k)$ required to 'cover' a generalized chessboard; the latter is represented by $R_{k}^{n}$, the set of n-vectors (or cells) with components in the ring of integers mod $k$. To explain the notion of 'cover' we first define the Hamming distance $\mathrm{d}_{H}(x, y)$ between two vectors ('squares' of the chessboard) as the number of components in which they differ; under the metric $d_{H}$, the board $R_{k}^{n}$ is a metric space. $R_{8}^{2}$ is the familiar chessboard. Then the rook domain or region covered by a rook at $x$ is the unit sphere $B(x, 1)=\left\{y \in R_{k}^{n} \mid d_{H}(x, y) \leq 1\right\}$.

Kamps and van Iint gave the following table of $\sigma(n, k)$ which represents almost all the known results to date for the above deterministic problem.

\section{Table 1}

$$
\text { Known Values of } \sigma(n, k)
$$

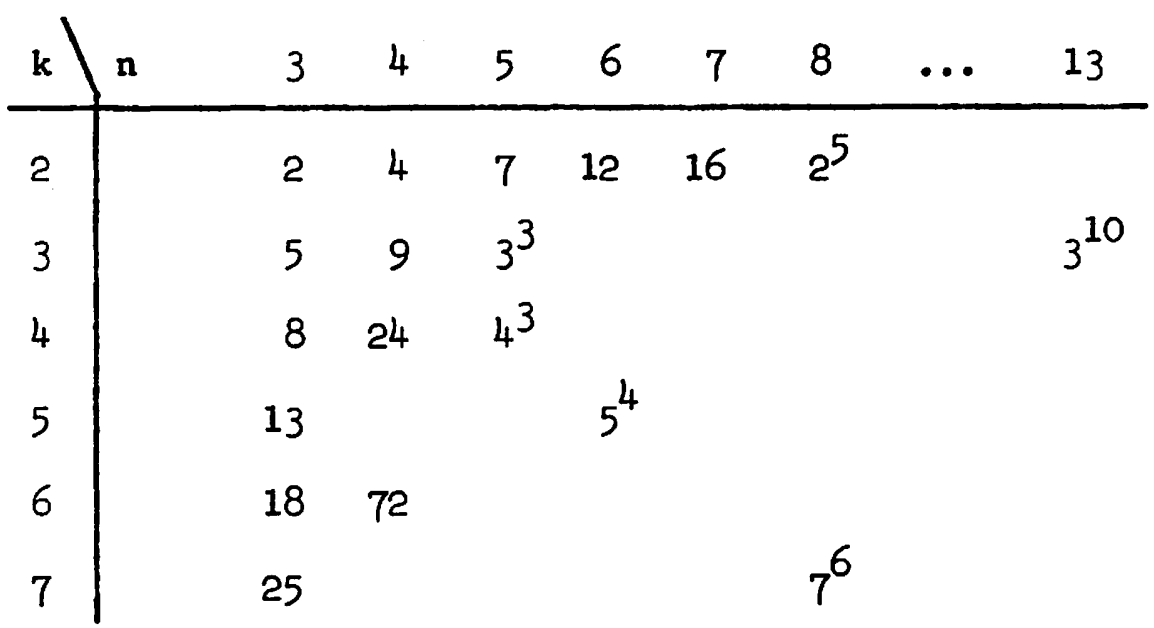

The only general results known (see their references) are 
(1) $\sigma(2, k)=k$,

(2) $\sigma(3, k)=\left[\left(k^{2}+1\right) / 2\right]$, where $[x]=$ integer part of $x$, and

(3) $\quad \sigma(n, k)=\frac{k^{n}}{1+n(k-1)}$, provided

(a) the right side of (3) is an integer (which implies $n>3$ ) and

(b) the integer $k$ is the power of a prime.

For example, from $(3) \quad \sigma(4,3)=q$ and from $(2)$, we have $\sigma(3,3) \neq 5$, Many values of $\sigma(n, k)$ were conputed by stanton $[4]$, stanton and Kalbfleisch $[5],[6]$ and others.

We consider two stochastic versions of the rook coverage problem. Rooks are placed in cells (vectors) sequentially and independently with uniform probabilities. We consider the distribution (in particular, the expectation) of the number of rooks $Y$ required to cover $R_{k}^{n}$ for the first time. In the multinomial case (Case $M$ ) the cells have constant probability $\mathrm{k}^{-\mathrm{n}}$ and repetition of occupancy is permitted. In the hypergeometric case (Case H) each successive occupancy is permitted only in the currently unoccupied cells, with uniform probability over these cells.

By introducing the stochastic version of the problem we feel that the problem has been broadened in an interesting and non-trivial manner. Indeed, although the deterministic problem is trivial for $n=2$, the corresponding stochastic problem is by no means trivial. Moreover it is hoped that the more general approach used in the stochastic version would lead to further extensions in the deterministic version, especially in the case of higher dimensions. Exact Solution for the Multinomial Case with $\mathbf{n}=2$.

Consider a 2-dimensional $k \times k$ chessboard. For Case $M$ let $Y_{M}$ denote the random number of rooks required to cover the $k \times k$ board 
and let $y$ denote values of $Y_{M^{*}}$ The event 'covering a row (column)' is equivalent to 'occupying a row (column)'.

Coverage of the board $R_{k}^{2}$ is characterized by occupancy either of all the rows or or all the columns. We also use the fact that for any given number of rooks, $N$, the number of rows occupied is independent of the number of columns occupied. Finally, occupancy of rows (similarly for columns) is a direct consequence of the classical Maxwe1l-Boltzmann statistics (see, for example, p. 59 of Feller [1]). In particular, the probability that all $k$ rows are occupied by $x$ randomly placed rooks is given exactly by

(4) $F_{k}(x)=\sum_{\alpha=0}^{k}(-1)^{\alpha}\left(\begin{array}{c}k \\ \alpha\end{array}\right)\left(1-\frac{\alpha}{k}\right)^{x}$

and the same result holds for columns. By virtue of the independence of row and colum occupancy, the cdf $G_{k}(y)$ of $Y_{M}$ is given by

(5) $G_{k}(y)=1-\left[1-F_{k}(y)\right]^{2}$.

The corresponding probability law $g_{k}(y)$ of $Y_{M}$ is obtained by taking differences in equation (5). Expectations are then obtained from $g_{k}(y)$ or by summing the complement of $G_{k}(y)$ over $y \geq 0$; this yields the two equivalent exact expressions

(6a) $E\left[Y_{m}\right]=k+\sum_{\beta=k}^{\infty}\left[\sum_{\alpha=1}^{k-1}(-1)^{\alpha}\left({ }_{\alpha}^{k}\right)\left(1-\frac{\alpha}{k}\right)^{\beta}\right]^{2}$

$$
=k+\frac{1}{k^{2(k-1)}} \sum_{i=1}^{k-1} \sum_{j=1}^{k-1}(-1)^{i+j} \frac{\left(\begin{array}{l}
k \\
i
\end{array}\right)\left(\begin{array}{l}
k \\
j
\end{array}\right)(i j)^{k}}{k^{2}-i j} \text {, }
$$

both of which are useful for computing (cf. Table 2). 
Exact Solution of the Hypergeometric Case for $\mathbf{n}=2$.

Here rooks are placed one-at-a-time independently and with uniform probability in the unoccupied cells. This case requires extensive modification of the solution strategy, mainly due to the loss of independence between row occupancies and column occupancies. We employ the method of inclusion-exclusion and Fréchet sums [1, p. 99] but the basic events have to be defined carefully.

First, we note that the $\mathrm{k}^{2}$-vector space (chessboard) is not covered by $y$ rooks if and only if at least one cell is not covered and this, in turn, holds if and only if at least one row is not oecupied and at least one column is not occupied. The event that a single cell is not covered, in positive terms, requires that all y rooks currently placed are in some $(k-1) \times(k-1)$ product subspace defined by the offending ce11. Intersections of these subspaces are again product subspaces, which may be indexed by the deleted rows and columns. Thus, we define our basic events $E_{i j}^{(y)}$ as the event frow $i$ and column $j$ are not covered when $y$ rooks are randomly placed\}. We now proceed to apply the Fréchet sum technique as follows.

In this hypergeometric set-up, y rooks can be placed without repetition in $\left(\begin{array}{l}k^{2} \\ y\end{array}\right)$ ways. They can fall in a subspace avoiding $r$ specified rows and $c$ specified columns in $\left(\begin{array}{c}(k-r)(k-c) \\ y\end{array}\right)$ ways and the probability of this event (not necessarily basic) is given by

$$
\left(\begin{array}{c}
(k-r)(k-c) \\
y
\end{array}\right) /\left(\begin{array}{l}
k^{2} \\
y
\end{array}\right)(y=0,1,2, \ldots)
$$

Since the $r$ rows and $c$ columns can be specified in $\left(\begin{array}{l}k \\ r\end{array}\right)\left(\begin{array}{l}k \\ c\end{array}\right)$ ways, the Fréchet sums, for a fixed total $t=r+c(r \geq 1, c \geq 1)$ of rows and colums not covered, are given by 
(8) $s_{t}(y)=\sum_{r=1}^{t-1}\left(\begin{array}{l}k \\ r\end{array}\right)\left(\begin{array}{c}k \\ t-r\end{array}\right)\left(\begin{array}{c}(k-r)(k-t+r) \\ y\end{array}\right) /\left(k_{y}^{k^{2}}\right)$.

According to the discussion above, if a cell is not covered then the sum $t$ of the rows and columns not covered is at least 2 and clearly $t \leq 2 k-y-1$. Hence the probability of realization of at least one of the basic events is

$$
\text { (9) } 1-H_{k}(y)=\sum_{t=2}^{2 k-y-1}(-1)^{t+1} s_{t}(y)
$$

where $H_{k}(y)$ is the cdf of the number of rooks required $Y_{H}$ for Case $H$. The expected value of $Y_{H}$ is obtained by summing (9) over $y \geq 0$. However the first $k$ terms are all equal to 1 . Since $Y_{H} \leq 1+(k-1)^{2}$, it follows that $1-H_{k}(y)=0$ for $y \geq 1+(k-1)^{2}$ and hence (10) $E\left[Y_{H}\right\}=k+\sum_{y=k}^{(k-1)^{2}}\left(1-H_{k}(y)\right)$.

This completes the exact solution for $E\left[Y_{H}\right]$ in Case H (cf. Table 2). Asymptotic Evaluations.

In Case $M$ we have from (4) asymptotically $(k \rightarrow \infty)$

$$
F_{k}(x)=\sum_{\alpha=0}^{k}(-1)^{\alpha}\left(\begin{array}{l}
k \\
\alpha
\end{array}\right)\left(1-\frac{\alpha}{k}\right)^{x}-\left(1-e^{-x / k}\right)^{k}-e^{-k e^{-x / k}} .
$$

Using the normalizing transformation [1, p. 106]

$$
\mathrm{x}=\mathrm{k} \ln \mathrm{k}+\mathrm{kz}
$$

we obtain for large $k$ the limiting cdf of $z$ (which takes on values $z$ )

(13) $v_{k}(z)=e^{-e^{-z}} \quad-\infty<z<\infty$,

the (standardized) extreme-value distribution. 
In our application $Y_{M}$ is the smaller of two independent chance variables each having the same cdf $F_{k}(x)$ and it follows from (12) that for $k \rightarrow \infty$

(14) $E\left[Y_{M}\right\}-k \ln k+k E\left\{z_{1: 2}\right\}=k(C+1 n k)$,

where $Z_{1: 2}$ is the smaller of 2 independent chance variables with cdf $V_{k}(z)$ in (13) and its expectation $C=-.1159315$ by the table of Lieblein and Salzer [3].

In Case $H$ we no longer have independence of row and column coverage and have to resort to an 'ad hoc method' to obtain a useful approximation which is as good as the approximation already obtained for Case M. Indeed one reason for considering the two cases together in the same paper is that we suspected that asymptotically the expectations for Case $M$ and Case $H$ would be the same to the first order approximation.

We make use of the fact that if we delete repetitions in placing rooks at random by the multinomial scheme, then the remaining observations $Y_{H}$ are formally indistinguishable from a hypergeometric sample sequence. The difference $D=Y_{M}-Y_{H}$ is the repetition in the multinomial sampling and our evaluation of $E\left\{Y_{H}\right\}$ arises by using

(15) $E\left\{Y_{H}\right\}=E\left\{Y_{M}\right\}-E\{D\}$.

To evalute $E[D]$ we first write $D=\sum_{i=1}^{k} \sum_{j=1}^{k} D_{i j}$, where $D_{i j}$ is the number of repetitions of extra rooks placed in the $(i, j)$ cell. The total number of rooks placed in the $(1, j)$ cell under multinomial sampling has a binomial distribution with parameters $Y_{M}$ and $1 / k^{2}$. Our 'ad hoc method' is to replace $Y_{M}$ by $E\left[Y_{M}\right\}$ in evaluating $E\left[D_{i j}\right\}$; 
we justify this by noting that the error introduced in the last expressions of $(16)$ and (17) below is of the order of magnitude $O\left(\frac{E\left[Y_{M}\right\}}{k^{2}}\right)=O\left(\frac{C+1 n k}{k}\right) \rightarrow 0$ as $k \rightarrow \infty$. We now obtain

(16) $E\left[D_{i j}\right] \sim \sum_{\alpha=2}^{E\left[Y_{M}\right\}}(\alpha-1)\left(_{\alpha}^{E\left[Y_{M}\right\}}\right)\left(\frac{1}{k^{2}}\right)^{\alpha}\left(1-\frac{1}{k^{2}}\right)^{E\left[Y_{M}\right\}-\alpha}$

$$
=\frac{1}{k^{2}} E\left(Y_{M}\right\}-1+\left(1-\frac{1}{k^{2}}\right)^{k(C+l n k)} \text {. }
$$

Using (14) for $E\left[Y_{M}\right\}$ and expanding the last term in (16) gives

(17) $E\left\{D_{i j}\right\}-\frac{1}{2}\left(\frac{C+1 n k}{k}\right)^{2}+o\left(\frac{1 n^{3} k}{k^{3}}\right)$

and the error term in (17) can also be disregarded. Thus, for the total set of $k^{2}$ cells we have from (17)

(18) $E\{D\}-\frac{1}{2}(C+\ln k)^{2}+o(1)$,

and hence by (15)

(19) $E\left\{Y_{H}\right\}-k(C+\ln k)-\frac{1}{2}(C+\ln k)^{2}$

where the error, which tends to zero as $k \rightarrow \infty$, is now omitted.

Table 2 gives exact values of $E\left[Y_{M}\right]$ for $k=2(1) 30$ using (6) and approximate values based on (14). It also gives exact values of $E\left\{Y_{H}\right\}$ for $k=2(1) 12$ using (10) and approximate values based on (19). Roundoff errors in this table are estimated to be at most one in the last digit shown.

Coverage of $\mathbf{k}^{\mathrm{n}}$-board for $\mathrm{n} \geq 2$.

Define a skeletal axis centered at cell $\mathrm{C}$ as the $\mathrm{n}$ mutually perpendicular lines of cells parallel to the sides of the hypercube and 
having the cell $\mathrm{C}$ in common; for $\mathrm{n}=3$ denote the cell by $\mathrm{c}_{\alpha, \beta, \gamma}$ $(\alpha, \beta, \gamma=1,2, \ldots, k)$ and the corresponding skeletal axis by $c^{\alpha, \beta, \gamma}$. For any $n$, a cell $c_{\alpha, \beta, \gamma}$ is not covered if and only if the skeletal axis $c^{\alpha, \beta, \gamma}$ has no occupancies. Hence we man use as our basic sets for an inclusion-exclusion argument the sets $c^{\alpha, \beta, \gamma}$ $(\alpha, \beta, \gamma=1,2, \ldots, k)$. However the intersections of these skeletal axes are not simple and the corresponding analysis is complicated even for $n=3$. A complete discussion of this analysis will not be considered here. Thus the stochastic problem becomes more difficult as $n$ increases as it does in the deterministic case of Kamps and van Lint [2]. Mr. Theodore Levy, a student of one of the authors at Michigan State University, is working on a class of such problems; the results are not yet very encouraging. Use of Independence in Higher Dimensions.

It is of some interest to find a way to generalize the independence of row occupancy and column occupancy that was used above for $n=2$. For this purpose we define a piece that starts at a cell $\mathrm{C}$ in $\mathrm{n}$ dimensions and moves (anywhere) inside any Hamming sphere centered at C and of radius $n-1$. For $n=2$ this reduces to the usual rook move. For $\mathrm{n}=3$ and starting at cell $\mathrm{C}$ the piece moves inside the horizontal plane (H-plane) through $\mathrm{C}$ or inside the north-south plane (NSplane) through $C$ or inside the east-west plane (EW-plane) through C. Hence one such piece covers all the cells in 3 mutually perpendicular slabs that contain the starting cell.

The cube $R_{k}^{3}$ will be covered as soon as either all $L$ slabs or all NS slabs or all EW slabs are occupied. Hence the same argument 
as for $n=2$ (Case $M$ ) gives for general $n$ (Case $M$ ) the exact solution for the cdf of $Y_{M}$

(20) $G_{k}(y)=1-\left[1-F_{k}(y)\right]^{n}$

where $F_{k}(y)$ is given by (4). For $n=3$ the expectation becomes

$\therefore i ; \quad E\left\{Y_{M}\right\}=k+\sum_{\beta=k}^{\infty}\left[\sum_{\alpha=1}^{k-1}(-1)^{\alpha}\left(\begin{array}{l}k \\ \alpha\end{array}\right)\left(1-\frac{\alpha}{k}\right)^{\beta}\right]^{3}$

$$
=k+\frac{1}{k^{3(k-1)}} \sum_{\alpha=1}^{k-1} \sum_{\beta=1}^{k-1} \sum_{\gamma=1}^{k-1}(-1)^{k-\alpha-\beta-\gamma} \frac{\left(\begin{array}{l}
k \\
\alpha
\end{array}\right)\left(\begin{array}{l}
k \\
\beta
\end{array}\right)\left(\begin{array}{l}
k \\
\gamma
\end{array}\right)(\alpha \beta \gamma)^{k}}{k^{3}-\alpha \beta \gamma},
$$

both of which can be used for computing.

In the corresponding asymptotic $(k \rightarrow \infty)$ evaluation for $n=3$ we need the expectation of the smallest of 3 independent observations on the cdf (13); this is given in [3] as -.4036136. This analysis is easily generalized to any number of dimensions $n$. This type of solution became possible only after we defined a 'super piece' that moved in more than one dimension. No similar analysis was found for the original definition of a rook move in the Haming sphere of radius 1. Acknowledgement.

The authors wish to acknowledge the assistance of Mr. Theodore Levy for his assistance in programming Table 1 and thanks are also due to Mr. Gary Simons of Stanford University for some preliminary deskcomputer computations. 
$\underline{\text { Table } 2}$

EXPECTED VALUES OF NUMBERS OF

RANDOM ROOKS REQUIRED TO COVER THE $\mathrm{k}^{2}$-CHESSBOARD

\begin{tabular}{|c|c|c|c|c|}
\hline $\mathbf{k}$ & $E\left\{Y_{M}\right\}$ & $\begin{array}{l}\text { Approximation to } \\
\qquad B\left\{Y_{M}\right\}\end{array}$ & $E\left\{Y_{M}\right\}$ & $\begin{array}{c}\text { Approximation to } \\
E\left(Y_{H}\right)\end{array}$ \\
\hline 2 & 2.3333333333 & 1.5544 & 2.0000000 & 1.3878 \\
\hline 3 & 4.1821428571 & 2.9480 & 3.5000000 & 2.4652 \\
\hline 4 & 6.3655677654 & 5.0815 & 5.3522478 & 4.2746 \\
\hline 5 & 8.7938685820 & 7.4675 & 7.4723892 & 6.3522 \\
\hline 6 & 11.4171670989 & 10.0550 & 9.8091916 & 8.6508 \\
\hline 7 & 14.2030879491 & 12.8099 & 12.3278253 & 11.1355 \\
\hline 8 & 17.1286506847 & 15.7081 & 15.0029299 & 13.7804 \\
\hline 9 & 20.1766249904 & 18.7316 & 17.8152024 & 16.5657 \\
\hline 10 & 23.3335906237 & 21.8665 & 20.7494692 & 19.4758 \\
\hline 11 & 26.5887915430 & 25.1016 & 23.7935002 & 22.4979 \\
\hline 12 & 29.9334107812 & 28.4277 & 26.9372363 & 25.6217 \\
\hline 13 & $33 \cdot 3600877782$ & 31.8372 & & 28.8384 \\
\hline 14 & 36.8625841610 & 35.3238 & & 32.1407 \\
\hline 15 & 40.4355447768 & 38.8818 & & 35.5223 \\
\hline 16 & 44.074322209 & 42.5065 & & 38.9776 \\
\hline 17 & 47.77484495 & 46.1938 & & 42.5020 \\
\hline 18 & 51.5335164 & 49.9399 & & 46.0911 \\
\hline 19 & 55.3471359 & 53.7416 & & 49.7414 \\
\hline 20 & 59.212836 & 57.5960 & & 53.4494 \\
\hline 21 & 63.12803 & 61.5004 & & 57.2121 \\
\hline 22 & 67.09038 & 65.4524 & & 61.0268 \\
\hline 23 & 71.09771 & 69.4499 & & 64.8910 \\
\hline 24 & 75.1481 & 73.4909 & & 68.8026 \\
\hline 25 & 79.2396 & 77.5736 & & 72.7595 \\
\hline 26 & 83.3704 & 81.6963 & & 76.7597 \\
\hline 27 & 87.539 & 85.8574 & & 80.8015 \\
\hline 28 & 91.743 & 90.0556 & & 84.8834 \\
\hline 29 & 95.981 & 94.2896 & & 89.0039 \\
\hline 30 & 100.250 & 98.5580 & & 93.1615 \\
\hline
\end{tabular}




\section{References}

[1] Feller, W. (1950). An Introduction to Probability Theory and its Applications, Volume 1. John Wiley and Sons, New York. Page references are to the third edition (1968).

[2] Kamps, H. J. L. and van Lint, J. H. (1970). A covering problem, to appear in a volume for the Colloquium on Combinatorial structures held at Balatonfured, Hungary, in August, 1969.

[3] Lieblein, J. and Salzer, H. E. (1957). Table of the first moment of ranked extremes. J. Res. Nat. Bur. Stand. $29 \underline{2}$ 203-206.

[4] Stanton, R. G. (1969). Covering theorems in groups (or: How to win at football pools). A paper included in Recent Progress in Combinatorics. Academic Press, New York.

[5] Stanton, R. G. and Kalbfleisch, J. G. (1968). Covering problems for dichotomized matchings. Aequat. Math. 1 94-103.

[6] Stanton, R. G. and Kalbfleisch, J. G. (1969). Intersection inequalities for the covering problem. Siam J. App1. Math. 17 1311-1316. 